\title{
Norois
}

Environnement, aménagement, société

\section{Atlas de la Vendée, Observatoire Économique, Social et territorial de la Vendée (OESTV) (éd.)}

La Roche-sur-Yon, 2004, 114 p.

\section{Jean Renard}

\section{OpenEdition}

\section{Journals}

Édition électronique

URL : http://journals.openedition.org/norois/703

DOI : $10.4000 /$ norois.703

ISBN : 978-2-7535-1542-0

ISSN : $1760-8546$

\section{Éditeur}

Presses universitaires de Rennes

\section{Édition imprimée}

Date de publication : 1 mars 2005

Pagination : 150-152

ISBN : 978-2-7535-0100-3

ISSN : 0029-182X

\section{Référence électronique}

Jean Renard, «Atlas de la Vendée, Observatoire Économique, Social et territorial de la Vendée (OESTV) (éd.) », Norois [En ligne], 194 | 2005/1, mis en ligne le 15 décembre 2008, consulté le 23 septembre 2020. URL : http://journals.openedition.org/norois/703 ; DOI : https://doi.org/10.4000/norois.703

Ce document a été généré automatiquement le 23 septembre 2020

(c) Tous droits réservés 


\section{Atlas de la Vendée, Observatoire Économique, Social et territorial de la Vendée (OESTV) (éd.)}

La Roche-sur-Yon, 2004, 114 p.

Jean Renard

\section{RÉFÉRENCE}

Atlas de la Vendée, 2004. - La Roche-sur-Yon, Observatoire Économique, Social et territorial de la Vendée (OESTV) (éd.) $114 \mathrm{p}$. 
1 L'atlas de la Vendée, réalisé par l'OESTV, sous la responsabilité de l'ancien sénateur J. Oudin qui anime depuis 1987 un observatoire avec une équipe de collaborateurs comprenant des géographes formés à l'université de Nantes, comble un vide. Il est le fruit d'une collaboration avec le Comité d'expansion économique disparu en 2003. Depuis notre manuel de géographie du département paru en 1978 (A. Chauvet, J. Renard, La Vendée, le pays et les hommes, éditions le Cercle d'Or, 246 p) il y a eu peu de documents cartographiques permettant de mesurer les évolutions de la géographie du département, si ce n'est ceux des services de l'État et des administrations. Seul le centre d'Études et
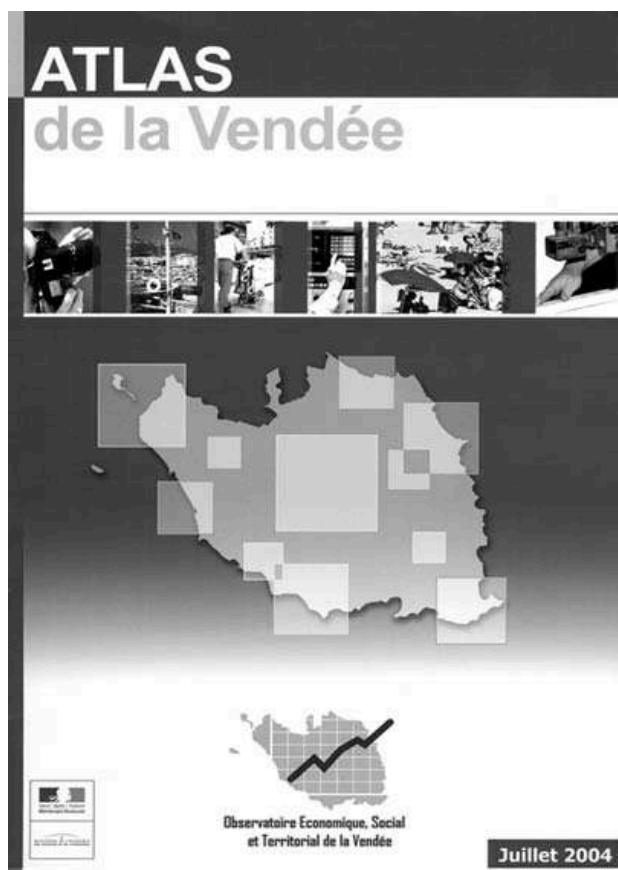
d'Action Sociale (CEAS) de Vendée publie régulièrement des cartes issues directement des données démographiques des recensements de population.

2 Nous disposons désormais, et c'est tant mieux, d'un bel atlas comportant de nombreuses cartes en couleur, dont il faut féliciter les auteurs pour le soin apporté à l'expression graphique et au choix des représentations. L'atlas est organisé en 14 chapitres couvrant les réalités économiques, démographiques et sociales du département. Il comprend 114 pages, et associe de brefs commentaires à une panoplie de cartes allant de l'échelle communale à l'ensemble de l'Ouest, mais aussi des courbes et des tableaux. Le choix des indicateurs retenus, l'utilisation judicieuse des couleurs et des signes proportionnels montrent le savoir faire des cartographes de l'observatoire, même si des scories demeurent (p. 95). Les traits originaux du département sont bien mis en valeur, notamment pour ce qui concerne la démographie et l'agriculture ou les indicateurs touristiques. Les données utilisées sont extraites des différents recensements de la population ou de l'agriculture, mais aussi des administrations du département, avec bon nombre de cartes décrivant les répartitions en 2003-2004, mais aussi les évolutions depuis un quart de siècle. En revanche les données sur les industries sont indigentes. L'atlas consacre 10 pages à l'agriculture, 3 à l'industrie, 2 au tourisme!

3 Le lecteur apprendra beaucoup sur un département aux territoires et aux dynamiques plus variés que ce que l'on a coutume de dire. À lire attentivement les documents on voit notamment se dessiner, au cœur du département, un pays yonnais jusqu'ici peu reconnu par bon nombre d'hommes politiques, mais qui s'affirme de plus en plus. Le sud du département quant à lui cumule les retards relatifs, et cela est dit, tandis que la dynamique du nord-est industriel n'est plus ce qu'elle était il y a encore 25 ans, notamment au plan démographique (p. 42-48). En revanche l'essor touristique du littoral est une réalité que la densité artisanale, le rythme des constructions, la multiplication des équipements, l'arrivée de retraités ou l'évolution des revenus des ménages soulignent. 
4 La carte des communautés de communes montre une couverture encore imparfaite, un petit nombre de communes rurales refusant de s'intégrer dans les nouveaux dispositifs. Une carte des pays au regard de la nouvelle politique régionale figure également, mais on se doit de regretter l'absence d'une carte de synthèse des territoires pertinents d'aménagement et de développement prenant en compte les espaces vécus des ménages. L'atlas en offre les possibilités et une telle réflexion ferait avancer la délimitation des pays de la loi Voynet, avec charte de territoire et conseil de développement.

5 Il s'agit, et cela est annoncé dès la première page, d'une première version. Chacun est invité à faire part de ses remarques et observations. Personnellement, et pour bien connaître le département, je souhaite que cet outil d'aide à la connaissance et à la décision, qu'il faut largement diffuser auprès des enseignants et de leurs élèves, s'enrichisse et devienne un atlas permanent. Un peu à la manière des observatoires de la dynamique des localisations, initiés par les géographes du GIP-Reclus de Montpellier à la fin des années 1980, et dont le laboratoire CESTAN, de l'institut de géographie de Nantes, a été un temps le correspondant pour l'Ouest de la France, en prenant mieux en compte tout ce qui bouge dans l'espace, et ce en temps réel.

6 Il faudrait donc élargir la palette des domaines à cartographier, y compris les données qui fâchent parce qu'elles ne sont pas politiquement correctes, ou qu'elles peuvent être sujet à polémique. L'atlas en donne quelques exemples, notamment à propos des cantons classés en zones d'excédent structurel ou à forte pression azotée. Je milite pour leur multiplication. Par exemple, et dans le désordre, on peut penser aux résultats des élections (politiques, syndicales, professionnelles); aux disparitions d'entreprises et aux créations sur le temps long ; aux évolutions des pratiques religieuses ; aux données sur l'évolution des taux de fécondité ; ou encore aux taux d'équipement des ménages (je pense à une carte par commune des ménages ayant deux voitures ou plus); ou encore les projets de localisation des lieux d'incinération des ordures ménagères; ou bien la part des actifs agricoles par commune; ou le rapport entre le nombre des exploitations professionnelles et le nombre de résidences secondaires. Mais encore la répartition des différentes catégories socio-professionnelles: où sont les ouvriers? Ou enfin la répartition des cabinets des professions libérales, des médecins spécialistes, des pharmacies et chirurgiens-dentistes, etc. Si la carte de la taille moyenne des exploitations agricoles est peu pertinente, en revanche l'apparition et la multiplication des très grandes exploitations a une signification forte. De même la répartition des exploitations ayant choisi de nouveaux modèles du type agriculture biologique, accueil à la ferme, ou réseau d'agriculture durable.

7 Un atlas territorial et social se doit de dépasser le traitement des données des seuls recensements et des statistiques banales et diversifier ses sources et indicateurs. Par exemple les données des Caisses d'allocations familiales sont d'une extraordinaire richesse. Leur traitement effectué dans l'Atlas social de l'agglomération nantaise par le CESTAN en montre l'intérêt. Mais ceci est également vrai pour les fichiers des permis de construire, les listes prud'homales, les taxes d'habitation permettant de connaître l'origine géographique des résidents secondaires, l'examen des résidences des patients reçus dans les hôpitaux ou des clients des grandes surfaces, etc. Le croisement des données, leur mise en perspective, et des analyses sur le temps long, en même temps que des informations en temps réel, sont désormais nécessaires pour mieux comprendre les dynamiques des territoires et dessiner les espaces de vie pertinents. 
8 Ces quelques remarques montrent l'intérêt rencontré à la lecture des cartes et des commentaires. Désormais, à l'ère de la cartographie assistée par ordinateur et de l'internet, et compte tenu de la masse des données disponibles, on doit espérer un partenariat entre tous les utilisateurs. Cela passe par une mise en commun des données, afin de disposer régulièrement, si possible en temps réel, des informations nécessaires sur les hommes et les lieux dans une optique résolument nouvelle. Je pense en particulier aux données de première main collectées dans le cadre des travaux universitaires et qui demeurent trop souvent confidentielles. Cet atlas est une première pierre à l'édifice et nous saluons sa parution dans l'attente de la prochaine édition.

\section{AUTEUR}

\section{JEAN RENARD}

IGARUN, Université de Nantes, Chemin de la Censive du Tertre, BP 81307, 44313 Nantes cedex 3, France,

renard.jean@numericable.fr 